\title{
Extended-Spectrum $\beta$-Lactamase-Producing Enterobacteriaceae: In vitro Susceptibility to Fosfomycin, Nitrofurantoin and Tigecycline
}

\author{
M. Al-Zarouni ${ }^{a} \quad$ A. Senok ${ }^{c} \quad$ N. Al-Zarooni ${ }^{a} \quad$ F. Al-Nassay ${ }^{a} \quad$ D. Panigrahi ${ }^{b}$ \\ ${ }^{a}$ AlQassimi Hospital Laboratory Sharjah, Ministry of Health, and ${ }^{b}$ Department of Clinical Sciences, College of \\ Medicine, University of Sharjah, Sharjah, United Arab Emirates; ' ${ }^{C}$ College of Medicine, Alfaisal University, \\ Riyadh, Saudi Arabia
}

\section{Key Words}

Extended-spectrum $\beta$-lactamase $\cdot$ Fosfomycin •

Tigecycline $\cdot$ Hyplex $\cdot$ Uropathogens

\begin{abstract}
Objective: To assess the susceptibility trends of communityacquired extended-spectrum $\beta$-lactamase (ESBL)-producing urinary isolates with particular reference to fosfomycin, nitrofurantoin and tigecycline. Materials and Methods: Seven hospitals across the United Arab Emirates participated in this study from June 2008 to March 2010. The antibiotic sensitivity of ESBL-producing uropathogens to a panel of antibiotics including tigecycline, fosfomycin and nitrofurantoin was assessed. The Hyplex ESBL identification system (h-ESID) was used for genotypic identification. Results: Two hundred and ninety-two ESBL-producing Enterobacteriaceae isolates were identified during the study period. Of these, $182(62 \%)$ were urinary isolates and comprised of Escherichia coli: 149 (81.9\%), Klebsiella pneumoniae: 30 (16.5\%) and Proteus mirabilis: 3 (1.6\%). Of the 182 urinary isolates, 179 (98.3\%) were from patients with community onset urinary tract infections. The h-ES-ID system identified 172 (94.5\%) of the urinary isolates as CTX-M positive. All isolates were susceptible to imipenem and meropenem. Over half of the isolates showed resistance to gentamicin $(98 ; 53.8 \%)$, trimethoprim-
\end{abstract}

sulfamethoxazole (139; 76.4\%) and ciprofloxacin (143; 78.6\%). Sensitivity to nitrofurantoin and fosfomycin was 90 and $100 \%$, respectively. Two CTX-M-positive K. pneumoniae isolates with tigecycline resistance (MIC $>4 \mu \mathrm{g} / \mathrm{ml}$ ) were identified. Conclusion: There is dissemination of CTX-M ESBL-producing urinary pathogens into the community. Fosfomycin and nitrofurantoin were active against ESBL-positive uropathogens, and emergence of tigecycline resistance needs close monitoring.

Copyright $\odot 2012$ S. Karger AG, Basel

\section{Introduction}

Pathogens producing extended-spectrum $\beta$-lactamases (ESBL) cause infections which are often associated with high mortality, morbidity and health care costs. The ESBL enzymes are commonly found in the Enterobacteriaceae family and over 120 ESBL types, including the CTX-M and OXA enzymes, have been described and they hydrolyze penicillins, broad-spectrum cephalosporins

This paper was presented in part as a poster at the 20th European Conference on Clinical Microbiology and Infectious Diseases (ECCMID), 10-13 April, 2010, Vienna, Austria.

\section{KARGER \\ Fax +41613061234 \\ E-Mail karger@karger.ch}

www.karger.com
(C) 2012 S. Karger AG, Basel

1011-7571/12/0216-0543\$38.00/0

Accessible online at:

www.karger.com/mpp
Dr. Abiola Senok

College of Medicine, Alfaisal University

PO Box 50927

Riyadh 11533 (Saudi Arabia)

Tel. +9661215 7661, E-Mail asenok@alfaisal.edu 
Table 1. Distribution of the total 292 isolates according to hospital

\begin{tabular}{lcc}
\hline & $\begin{array}{l}\text { Total number } \\
\text { of isolates }\end{array}$ & $\begin{array}{l}\text { Number of } \\
\text { urinary isolates }\end{array}$ \\
\hline Al Qassimi Hospital, Sharjah & 79 & 29 \\
Kuwaiti Hospital, Sharjah & 29 & 21 \\
Um Al Quwain Hospital & 7 & 3 \\
Sheikh Khalifa Hospital, Ajman & 11 & 10 \\
Al Wasel Hospital, Dubai & 8 & 4 \\
Dubai Hospital & 127 & 99 \\
Al Rashid Hospital, Dubai & 31 & 16 \\
\hline Total & 292 & 182 \\
\hline
\end{tabular}

and monobactams [1]. However, they do not affect cephamycins and carbapenems, and they are inhibited by clavulanic acid [1].

Currently, the emergence and rapid dissemination of CTX-M-positive ESBL-producing bacteria have caused a change in ESBL epidemiology [1-3]. As these ESBL-producing strains often exhibit a multidrug-resistance phenotype, the increase in community-acquired urinary tract infections (UTIs) caused by CTX-M ESBL producers poses significant challenges for the empiric treatment of these infections in the outpatient setting. Furthermore, the recent identification of ESBL-producing isolates that have acquired carbapenemases has further limited the therapeutic options available for treatment of these multidrug-resistant microorganisms [4]. This scenario has resulted in the need for reevaluating the efficacy of old antimicrobials such as nitrofurantoin and fosfomycin for their activity against these community-acquired ESBLproducing uropathogens. In addition, there is growing interest in the evaluation of new-generation antibiotics such as tigecycline for treating nosocomial infections caused by ESBL-producing isolates. In this report, the antibiotic susceptibility trends of community-acquired ESBL-producing urinary isolates in the United Arab Emirates are presented - particularly, the in vitro activity of tigecycline, fosfomycin and nitrofurantoin against these organisms.

\section{Materials and Methods}

Two hundred and ninety-two ESBL-producing Enterobacteriaceae comprised of Escherichia coli $(\mathrm{n}=225)$, Klebsiella pneumoniae ( $\mathrm{n}=60)$, Proteus mirabilis $(\mathrm{n}=4)$, Enterobacter cloacae $(\mathrm{n}=2)$ and Acinetobacter baumanii $(\mathrm{n}=1)$ were obtained from participating hospitals as shown in table 1 . Of the 292 isolates, 182 were urinary isolates and these were included for further study of antibiotic susceptibility testing and genotyping using the Hyplex ESBL identification system (h-ES-ID). Seven hospitals across the United Arab Emirates (UAE) participated in this study, which was carried out from June 2008 to March 2010. Nonduplicated ESBLpositive Enterobacteriaceae obtained from the participating hospitals were stored in $20 \%$ glycerol at $-20^{\circ} \mathrm{C}$ and sent to the Microbiology Laboratory, Al-Qassimi Hospital, Sharjah, UAE, and reconfirmation of the ESBL phenotype was carried out.

\section{Identification of ESBL Phenotype}

ESBL screening was carried out using the VITEK II (bioMérieux, Marcy l'Etoile, France) and MicroScan Walk-Away (Siemens Healthcare Diagnostics, Sacramento, Calif., USA) automated systems. Manual screening of isolates was also done by disk diffusion using ceftazidime $(30 \mu \mathrm{g})$ and cefpodoxime (10 $\mu \mathrm{g})$ according to the Clinical and Laboratory Standards Institute's (CLSI) recommended guidelines [5]. Confirmation of ESBL phenotype was carried out by using the ESBL E-test according to the manufacturer's guidelines. E. coli ATCC 51446 and K. pneumoniae ATCC 700603 were used as positive controls and E. coli ATCC 25922 as negative control.

\section{Determination of ESBL Genotype Using the h-ES-ID \\ Multiplex PCR-ELISA System}

The multiplex PCR and reverse-hybridization steps were carried out using the reagents and instructions provided by the manufacturer. Control reactions for evaluating the PCR and hybridization steps were performed in each run. For the identification of isolates with the $b l a_{\mathrm{CTX}-\mathrm{M}}$ gene, a CTX-M-specific probe was used. The multiplex PCR amplification was performed using $2 \mu \mathrm{l}$ of the primer mix, $1 \mu \mathrm{l}$ of nucleotide mix of the h-ES-ID PCR module and $5 \mu$ l of the sample supernatant, $5 \mu$ l of $10 \times$ PCR buffer, $1 \mu \mathrm{l}$ UltraStart DNA polymerase and $50 \mu \mathrm{l}$ of double-distilled water. The cycling conditions were as follows: initial denaturation step at $94^{\circ} \mathrm{C}$ for $5 \mathrm{~min}, 35$ cycles of denaturation at $94^{\circ} \mathrm{C}$ for $25 \mathrm{~s}$, annealing at $52^{\circ} \mathrm{C}$ for $25 \mathrm{~s}$, elongation at $72^{\circ} \mathrm{C}$ for $20 \mathrm{~s}$ with increasing $1 \mathrm{~s}$ per cycle and a final extension of $3 \mathrm{~min}$ at $72^{\circ} \mathrm{C}$. The amplification products were stored at $-20^{\circ} \mathrm{C}$. For reverse hybridization, amplification products were heated at $95^{\circ} \mathrm{C}$ for $10 \mathrm{~min}$ and then mixed with the hybridization buffer. Aliquots of this mixture were added to color-coded micro-well plate cavities adsorbed with the consensus probe for TEM, SHV, CTX-M and OXA (to confirm ESBL positivity) as well as a CTX-M-specific probe for identification of CTX-M genotype. Plates were incubated at $50^{\circ} \mathrm{C}$ for $30 \mathrm{~min}$ for hybridization and then incubated with $100 \mu l$ peroxidase conjugate per well for $30 \mathrm{~min}$ at room temperature and washed. Incubation with $100 \mu$ l of substrate solution was carried out and the reaction was terminated by addition of stop solution. Extinction measurement was done within $60 \mathrm{~min}$ of termination using a photometer at $450 \mathrm{~nm}$ and a reference length of 620-650 $\mathrm{nm}$. Zero comparison was made against air and samples with extinction values $>400$ were considered positive, 200-400 borderline and $<200$ negative.

\section{Antibiotic Susceptibility Testing}

Susceptibility of the isolates to various antimicrobial agents was determined by the Kirby-Bauer disk diffusion technique on Mueller-Hinton agar plates as previously described [6]. The following antibiotics were tested for all isolates: meropenem $(10 \mu \mathrm{g})$, 
imipenem $(10 \mu \mathrm{g})$, amikacin $(30 \mu \mathrm{g})$, gentamicin $(10 \mu \mathrm{g})$, piperacillin/tazobactam $(110 / 10 \mu \mathrm{g})$, ciprofloxacin $(5 \mu \mathrm{g})$ and tigecycline $(15 \mu \mathrm{g})$. The sensitivity of urinary isolates to nitrofurantoin $(300 \mu \mathrm{g})$ and trimethoprim-sulfamethoxazole $(1.25 / 23.75 \mu \mathrm{g})$ was determined. Susceptibility to fosfomycin was assessed for 114 urinary isolates (92 E. coli and $22 \mathrm{~K}$. pneumoniae). The results were recorded and interpreted according to CLSI criteria. Determination of MIC with the E-test was carried out for meropenem and tigecycline according to the manufacturer's guidelines as previously described [6].

Statistical analysis using the $\chi^{2}$ test was performed using SigmaStatver 3.5 software (Systat Software Inc., San Jose, Calif., USA). $\mathrm{p}<0.05$ was considered statistically significant.

\section{Results}

The 182 urinary isolates studied comprised 149 (81.9\%) E. coli, 30 (16.5\%) K. pneumoniae and 3 (1.6\%) P. mirabilis . Of these 182 urinary isolates, 179 (98.3\%) were from patients with community onset UTIs, and the ESBL-positive isolates were correctly identified using automated methods, i.e. screening by disk diffusion, ESBL E-test and the h-ES-ID. Using the $b l a_{\text {CTX-M }}$ specific probe of the hES-ID system, 172 (94.5\%) were found to be CTX-M positive while the remaining 10 (5.5\%) were CTX-M-negative; these include E. coli $(\mathrm{n}=7) ; K$. pneumoniae $(\mathrm{n}=2)$ and $P$. mirabilis $(\mathrm{n}=1)$. Of the 182 urinary isolates studied, $163(90 \%)$ were susceptible to nitrofurantoin while $114(62.6 \%)$ community onset urinary isolates were susceptible to fosfomycin; these include E. coli $(\mathrm{n}=93)$, and $K$. pneumonia $(\mathrm{n}=21)$. As there are no accepted breakpoints for K. pneumoniae, those proposed by CLSI for $E$. coli were used. All 114 isolates were susceptible to fosfomycin with a mean inhibition zone diameter of $36.8 \pm$ $7.0 \mathrm{~mm}$ (range 19-57). Sensitivity to nitrofurantoin and fosfomycin was significantly higher compared with gentamicin, ciprofloxacin and trimethoprim-sulfamethoxazole $(\mathrm{p}<0.001)$.

Two (1.1\%) CTX-M-positive K. pneumoniae isolates showed resistance to tigecycline with an MIC $>4 \mu \mathrm{g} / \mathrm{ml}$. These isolates were from adult female patients with community onset UTIs.

All isolates were sensitive to imipenem and meropenem. Of the 182 isolates, 175 (96\%) were sensitive to amikacin and 172 (95\%) were sensitive to piperacillin-tazobactam (fig. 1). In contrast, over half of the isolates showed resistance to gentamicin 98 (53.8\%), trimethoprim-sulfamethoxazole 139 (76.4\%) and ciprofloxacin 143 (78.6\%) (fig. 1). Ciprofloxacin resistance was $80 \%$ among CTXM-positive isolates compared to $37.5 \%$ in CTX-M-negative isolates.

Antibiotic Susceptibility of ESBL-Positive Uropathogens

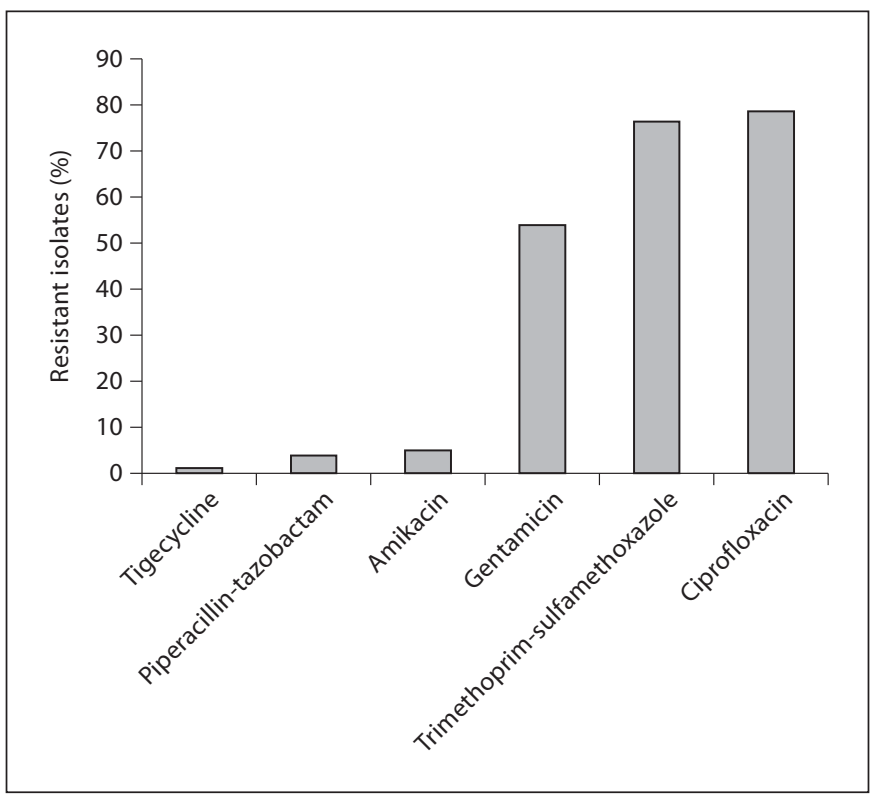

Fig. 1. Antibiotic resistance pattern of ESBL-positive urinary isolates.

\section{Discussion}

In this study, the majority of ESBL-producing Enterobacteriaceae identified were urinary isolates associated with community onset UTIs. These urinary isolates were highly resistant to ciprofloxacin and trimethoprim-sulfamethoxazole, the commonly used antibiotics in the outpatient setting for treatment of UTIs. Although they remained predominantly sensitive to carbapenems (imipenem and meropenem), amikacin and piperacillintazobactam, these are parenterally administered and reserved for inpatient use. Our findings demonstrated fosfomycin susceptibility in all 114 isolates tested. Fosfomycin is a cell wall inhibitor whose broad spectrum of activity, therapeutic range and oral administration make it pertinent for use in the treatment of UTIs in the outpatient setting [7]. Although E. coli rapidly develops resistance to fosfomycin, experimental in vitro studies have shown that resistant isolates have lowered fitness and poor survival [7]. Hence, fosfomycin remains active against many common uropathogens, including ESBL producers. A recent report has also described good activity of fosfomycin against carbapenem-resistant Enterobacteriaceae, including NDM-1-positive isolates [8]. In a survey of 428 ESBL-producing isolates in Spain, fosfomycin resistance was 0.3 and $7.2 \%$ in E. coli and K. pneumoniae, respectively [9]. In Brazil, where there is high us- 
age of fosfomycin, up to $98.8 \%$ of E. coli isolates from community-acquired UTIs were fosfomycin sensitive [10]. Our findings of $100 \%$ susceptibility among the ESBL-producing isolates tested provide further supportive evidence for recommending fosfomycin for the management of community acquired ESBL-producing urinary isolates.

Nitrofurantoin is a synthetic nitrofuran antimicrobial agent which has been used for over 50 years in the outpatient setting for the treatment of uncomplicated UTIs. In this study, $90 \%$ of the isolates were susceptible to nitrofurantoin. Varying levels of nitrofurantoin sensitivity in urinary ESBL isolates have been reported in the literature. Whilst high sensitivity levels have been reported from surveys in the USA ( $>88 \%)$, India (89\%) and Hong Kong (98\%), reports of lower sensitivity levels have emerged from Italy, Latin America and France [7, $11,12]$. Compared with these surveys, the $90 \%$ susceptibility to nitrofurantoin reported in this study is relatively high and similar to that reported data from Kuwait [13]. In addition, it is also significantly higher compared to data from neighboring countries in the region, i.e. 73 and $61.8 \%$ of urinary isolates from Saudi Arabia and Bahrain, respectively, were reported to be sensitive to nitrofurantoin $[14,15]$. These findings indicate that both nitrofurantoin and fosfomycin represent alternative therapeutic agents for the treatment of community onset UTIs caused by ESBL-producing uropathogens in our setting.

Tigecycline is a glycylcycline derivative of minocycline with broad-spectrum activity against Gram-positive and Gram-negative bacteria. Tigecycline is not affected by the presence of coresistance mechanisms in unrelated antimicrobials, thus representing an attractive alternative in ESBL isolates which often exhibit other re- sistance phenotypes. Two K. pneumoniae isolates with elevated tigecycline MICs were identified, suggestive of the emergence of isolates resistant to this antibiotic. Similar resistance to tigecycline has recently been reported by Livermore et al. [8] among carbapenemase-producing Klebsiella and Enterobacter isolates in the United Kingdom. Although these findings indicate that tigecycline remains useful for isolates circulating in our setting, the implementation of good antibiotic stewardship and active surveillance is recommended particularly in the community and outpatient setting.

Compared with other reports from the Arabian Gulf region, the findings in this study demonstrate a significantly higher frequency of bla $a_{\text {CTX-M }}$ ESBL-positive isolates. In recent reports from the UAE and Kuwait, the proportions of Enterobacteriaceae isolates with the CTXM genotype were 87 and $77.9 \%$, respectively $[16,17]$. Lower figures have emerged from Saudi Arabia, with two recent reports showing bla $a_{\mathrm{CTX}-\mathrm{M}}$ detection in 34 and $71 \%$ of Enterobacteriaceae isolates $[14,18]$. In an earlier report, ESBL producers were identified mainly as agents of nosocomial infection in the UAE [6]. These new findings are of particular concern as they indicate that there is a trend towards increasing dissemination of CTX-M ESBL isolates into the community.

\section{Conclusion}

Our findings have demonstrated a high level of CTXM ESBLs with dissemination into the community. Further, both nitrofurantoin and fosfomycin were effective therapeutic agents in the treatment of community onset UTIs caused by ESBL-positive pathogens.

\section{References}

1 Paterson DL, Bonomo RA: Extended-spectrum beta-lactamases: a clinical update. Clin Microbiol Rev 2005; 18:657-686.

$\checkmark 2$ Livermore DM, Hawkey PM: CTX-M: changing the face of ESBLs in the UK. J Antimicrob Chemother 2005;56:451-454.

$>3$ Pitout JD, Laupland KB: Extended-spectrum beta-lactamase-producing Enterobacteriaceae: an emerging public-health concern. Lancet Infect Dis 2008;8:159-166.
-4 Nordmann P, Poirel L, Toleman MA, Walsh TR: Does broad-spectrum beta-lactam resistance due to NDM-1 herald the end of the antibiotic era for treatment of infections caused by Gram-negative bacteria? J Antimicrob Chemother 2011;66:689-692.

5 National Committee for Clinical Laboratory Standards: Performance Standards for Antimicrobial Susceptibility Testing. Wayne, National Committee for Clinical Laboratory Standards (CLSI), 2005, 15th Informational Supplement (m100-s15).
6 Al-Zarouni M, Senok A, Rashid F, Al-Jesmi SM, Panigrahi D: Prevalence and antimicrobial susceptibility pattern of extended-spectrum beta-lactamase-producing Enterobacteriaceae in the United Arab Emirates. Med Princ Pract 2008; 17:32-36.

7 Garau J: Other antimicrobials of interest in the era of extended-spectrum beta-lactamases: fosfomycin, nitrofurantoin and tigecycline. Clin Microbiol Infect Dis 2008; 14(suppl 1):198-202. 
$>8$ Livermore DM, Warner M, Mushtaq S, Doumith M, Zhang J, Woodford N: What remains against carbapenem-resistant Enterobacteriaceae? Evaluation of chloramphenicol, ciprofloxacin, colistin, fosfomycin, minocycline, nitrofurantoin, temocillin and tigecycline. Int J Antimicrob Agents 2011;37: 415-419.

$>9$ de Cueto M, Hernandez JR, Lopez-Cerero L, Morillo C, Pascual A: Activity of fosfomycin against extended-spectrum beta-lactamase producing Escherichia coli and Klebsiella pneumoniae. Enferm Infecc Microbiol Clin 2006;24:613-616.

$>10$ Biondo CM, Rocha JL, Tuon FF: Fosfomycin in vitro resistance of Escherichia coli from the community. Braz J Infect Dis 2011;15:96.
Zhanel GG, Hisanaga TL, Laing NM, DeCorby MR, Nichol KA, Weshnoweski B, Johnson J, Noreddin A, Low DE, Karlowsky JA, Hoban DJ: Antibiotic resistance in Escherichia coli outpatient urinary isolates: final results from the North American Urinary Tract Infection Collaborative Alliance (NAUTICA). Int J Antimicrob Agents 2006; 27:468-475.

12 Fadda G, Nicoletti G, Schito GC, Tempera G: Antimicrobial susceptibility patterns of contemporary pathogens from uncomplicated urinary tract infections isolated in a multicenter Italian survey: possible impact on guidelines. J Chemother 2005; 17:251-257.

13 Al Benwan K, Al Sweih N, Rotimi VO: Etiology and antibiotic susceptibility patterns of community- and hospital-acquired urinary tract infections in a general hospital in $\mathrm{Ku}$ wait. Med Princ Pract 2010;19:440-446.

14 Bindayna K, Senok A, Jamsheer A: Extended Spectrum Beta-lactamase-producing Enterobacteriaceae in Bahrain: Prevalence and antibiotic sensitivity pattern. J Infect Public Health 2009;2:129-135.
5 Khanfar HS, Bindayna KM, Senok AC, Botta GA: Extended spectrum beta-lactamases (ESBL) in Escherichia coli and Klebsiella pneumoniae: trends in the hospital and community settings. J Infect Dev Ctries 2009;3: 295-299.

16 Alfaresi MS, Elkoush AA, Alshehhi HM, Abdulsalam AI: Molecular characterization and epidemiology of extended-spectrum beta-lactamase-producing Escherichia coli and Klebsiella pneumoniae isolates in the United Arab Emirates. Med Princ Pract 2011;20: 177-180.

17 Al Hashem G, Al Sweih N, Jamal W, Rotimi VO: Sequence analysis of $b l a_{\text {CTX-M }}$ genes carried by clinically significant Escherichia coli isolates in Kuwait hospitals. Med Princ Pract 2011;20:213-219.

18 Al-Agamy MH, Shibl AM, Tawfik AF: Prevalence and molecular characterization of extended-spectrum beta-lactamase-producing Klebsiella pneumoniae in Riyadh, Saudi Arabia. Ann Saudi Med 2009;29:253-257. 